\title{
Investigating Artefacts and Repair Mechanisms Associated with $\alpha$-particle Interactions in Charge Coupled Devices
}

\author{
Rosie Newton $^{1}$, Mike J. Scott ${ }^{2}$, and Malcolm J. Joyce ${ }^{1}$ \\ ${ }^{1}$ Lancaster University, United Kingdom \\ ${ }^{2}$ BIC Technology Ltd., United Kingdom
}

\begin{abstract}
Charge coupled devices (CCDs) have been shown to have potential for detecting charged particles and other forms of ionising radiation. In particular, the clusters in the pixel images produced are distinctive for $\alpha$ and $\beta$ radiations, with $\alpha$ particles causing large, symmetrical clusters or long, vertical tracks often referred to as blooming, and $\beta$ particles causing long, curved tracks. The size and shape of these tracks are also related to the energy of the incident radiation, giving the potential for spectroscopy of these types of radiation. This could be used, for example, to realise a hand-held, portable device for in-situ detection and identification of radioactive contamination. Images have been taken of exposures to a ${ }^{210} \mathrm{Po} \alpha$-particle source using Sony ICX825AL interline transfer CCDs, covered with a thin, aluminised Mylar film to prevent inadvertent exposure to light. Both vertical streaks and round clusters have been observed in each of the three CCDs used. Increased levels of noise have been seen after several hours of exposure to the ${ }^{210} \mathrm{Po}$ source, found to be caused by damage from $\alpha$ radiation. Three methods have been investigated to reduce this noise: 1) Annealing at $100^{\circ} \mathrm{C}$ for 24 hours repairs a significant proportion of the damage caused by the $\alpha$ radiation, though the effectiveness is reduced with each subsequent exposure and annealing cycle; 2) Cooling is performed during exposures by using a heat pipe to move heat away from the back of the CCD and a fan to cool the heat pipe and electronics associated with the CCD. This reduces the dark current during CCD measurements, and the method of cooling used allows the device to retain its small, hand-held size; 3) Any remaining noise can be removed later using image processing. These techniques are combined to extend the lifetime of the device. The rate of damage from the ${ }^{210} \mathrm{Po}$ source is similar whether the device is cooled or not, however the noise evident in the images produced when cooled is significantly less, allowing the $C C D$ to be used for a longer time between annealing operations.
\end{abstract}

Index Terms-Charge coupled device, alpha radiation, radiation damage.

\section{INTRODUCTION}

THE detection and identification of radioactive 1 contamination is essential in the nuclear industry but may be difficult to achieve in-situ for $\alpha$ radiation, owing to its short range in air. Because $\alpha$-emitting radioactivity is particularly hazardous if inhaled or ingested, identifying $\alpha$ contamination is critical to managing this risk. Usually, $\alpha$-particle spectroscopy is performed in a laboratory by processing the sample to isolate the radioisotope and detecting the $\alpha$ radiation under a vacuum. This procedure is not always practical because of the processing and equipment involved.

CCDs are small, semiconductor pixel-imaging devices commonly used in digital cameras. Different types of radiation leave distinctive tracks in CCDs, allowing them to be used for identification of radiation. CCDs are small in size making them suitable for use in a hand-held instrument, and potentially usable in-situ.

As radiation enters a $\mathrm{CCD}$, charge carriers are created via ionization in the active region of the device, as shown in Fig. 1. The evolved charge is then collected by gate electrodes, passed through adjacent pixels, and read out sequentially to form a pixelated image based on the location of the charge carrier distribution in the $\mathrm{CCD}$, and hence that of the associated interactions. Different types of artifacts may constitute these images, depending on the interactions of the radiation in the $\mathrm{CCD}$. As the radiation scatters through several pixels, this will leave a trail of charge carriers, resulting in a track of pixels. If a large amount of energy is deposited in a given pixel, it diffuses through the substrate to create large, symmetrical clusters of pixels. If the amount of created charge reaches the capacity of the pixel, it may overflow vertically. This "blooming" would be expected to leave a vertical streak of pixels in the absence of any blooming correction facilities.
Manuscript received July 11, 2019. This work was supported by the Next Generation Nuclear Centre for Doctoral Training, the Engineering and Physical Sciences Research Council (EPSRC), and the Lloyds Register Foundation. M. J. Joyce acknowledges the support of the Royal Society as a recipient of a Wolfson Research Merit award.
Rosie Newton and Malcolm J. Joyce are with the Engineering Department, Lancaster University, LA1 4YW, UK. (e-mail: r.newton1@1ancaster.ac.uk).

Mike J. Scott is with BIC Technology Ltd., Gostin House, 32/36 Hanover St., Liverpool, L1 4LN, UK (e-mail: info@bictechnology.co.uk) 


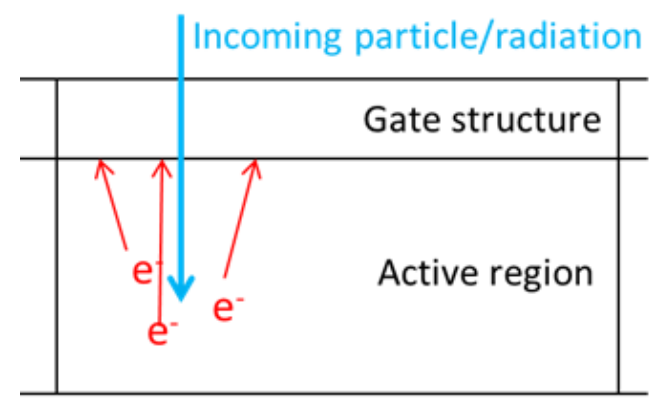

\section{Substrate}

Fig. 1. A schematic cross section of a single pixel within a CCD.

\section{PRIOR ART}

It has been reported that $\beta$ particles often cause long, curved tracks as they scatter through the CCD, and that $\alpha$ particles typically form large, round clusters [1], [2]. This is because the latter interact more strongly, creating a larger number of charge carriers in a single pixel which diffuses into the surrounding pixels. Occasionally, $\alpha$ particles will also bloom into a vertical streak.

In research prior to that presented here, similar differences have been seen, as shown in Fig. 2. Here, the larger round clusters and vertical streaks from $\alpha$ radiation can be seen as a result of exposure to a ${ }^{210} \mathrm{Po}$ source. In the exposures to ${ }^{60} \mathrm{Co}$ and ${ }^{137} \mathrm{Cs}$, smaller clusters and curved tracks can be seen from the $\beta$ and $\gamma$ radiation. These can be used to identify $\alpha$ and $\beta$ sources, respectively, through the analysis of the sizes and shapes of the clusters produced [3], [4].
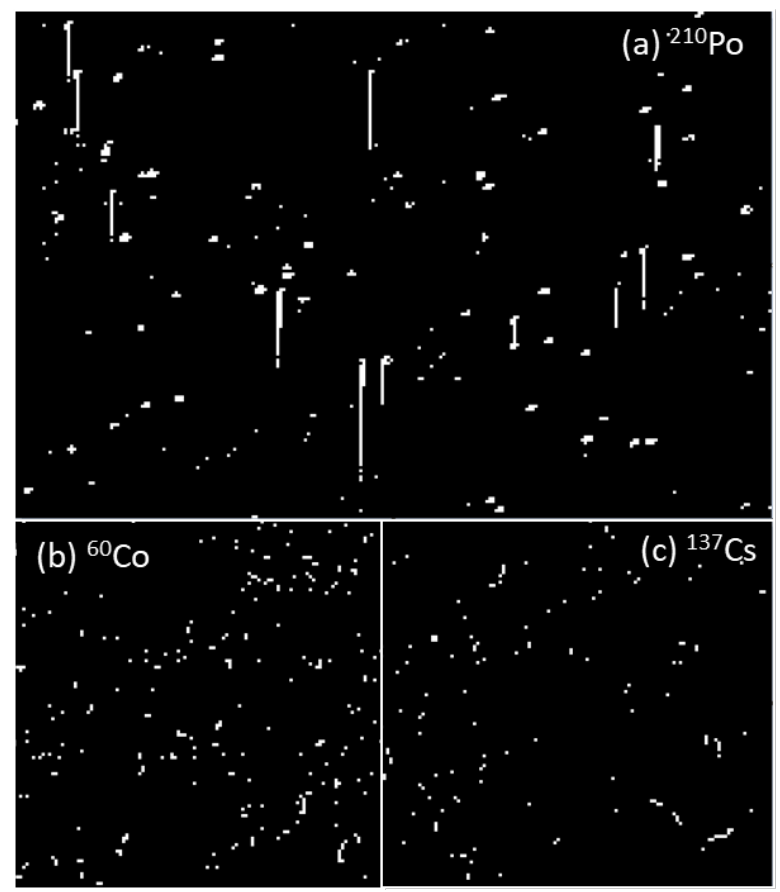

Fig. 2. Example sections of typical exposures to: (a) a ${ }^{210} \mathrm{Po} \alpha$-particle source for $30 \mathrm{~s}$; (b) a ${ }^{60} \mathrm{Co} \beta / \gamma$ source for $5 \mathrm{~s}$; (c) a ${ }^{137} \mathrm{Cs} \beta / \gamma$ source for $1 \mathrm{~s}$.

\section{CCD DETAILS}

The CCD used for this research is a Sony ICX825AL. This is an interline CCD with $1392 \times 1040$ pixels and a pixel size of $6.45 \times 6.45 \mu \mathrm{m}$, for a total imaging area of $6.71 \times 8.98 \mathrm{~mm}$.
The device can be seen in Fig. 3, with the CCD itself covered with an aluminised Mylar film to prevent the interaction of light which would otherwise dominate the image. The CCD can be seen uncovered in the inset image.

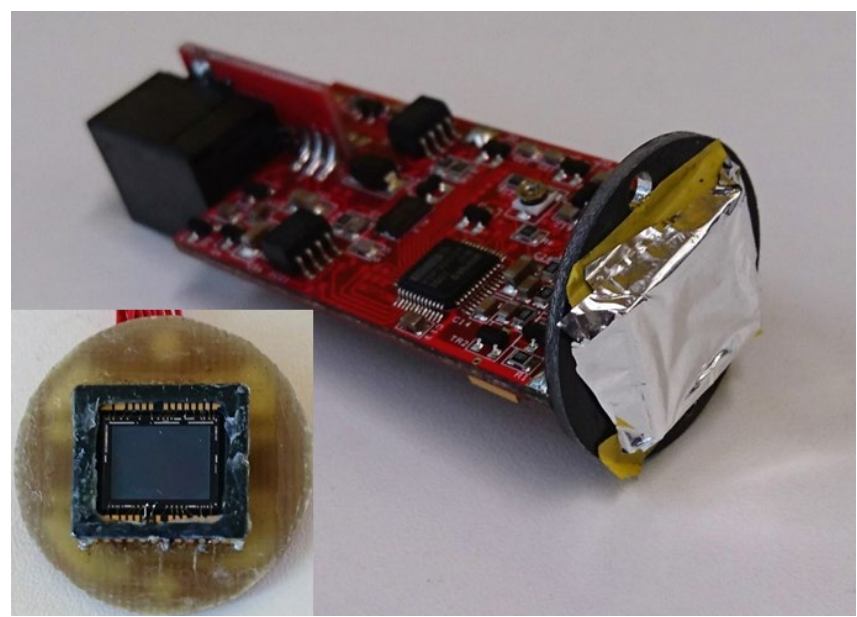

Fig. 3. $\alpha$-particle detection device used in this research showing the CCD covered with Mylar film and the associated electronics. Inset: Uncovered CCD.

\section{Alpha Damage}

Over continued exposure of the CCD to $\alpha$ radiation, an increase in noise was observed in the images produced. This noise consists of bright pixels where no interactions with radiation have occurred during the current exposure. At low levels of noise this does not cause a significant problem, however a high level of noise can impact the accuracy of $\alpha$ particle detection. To confirm that the noise was caused by damage from the $\alpha$ radiation, half of the CCD imaging area was covered with card sufficiently thick to stop the $\alpha$ particles. The CCD was then exposed to a ${ }^{210} \mathrm{Po}$ source with an activity of $21 \mathrm{~Bq}$ for five hours. At each hour interval, a background measurement was taken with no source present. Consistent sections of each side of the images produced were then analysed to identify a difference in the number of signals in the background between the covered and uncovered sides of the CCD. The data can be seen in Fig. 4.

It is clear that the uncovered section which has been exposed to the $\alpha$ radiation has increased in background signals with each additional hour of exposure. In contrast, the covered side which prevented $\alpha$ particles from interacting with the CCD showed no change over the 5-hour period. Similar experiments were performed with the $320 \mathrm{kBq}{ }^{137} \mathrm{Cs}$ source and, over a period of 9 hours, no change was seen in the background. This indicates that $\alpha$ radiation is the most significant cause of the increase in background noise. 


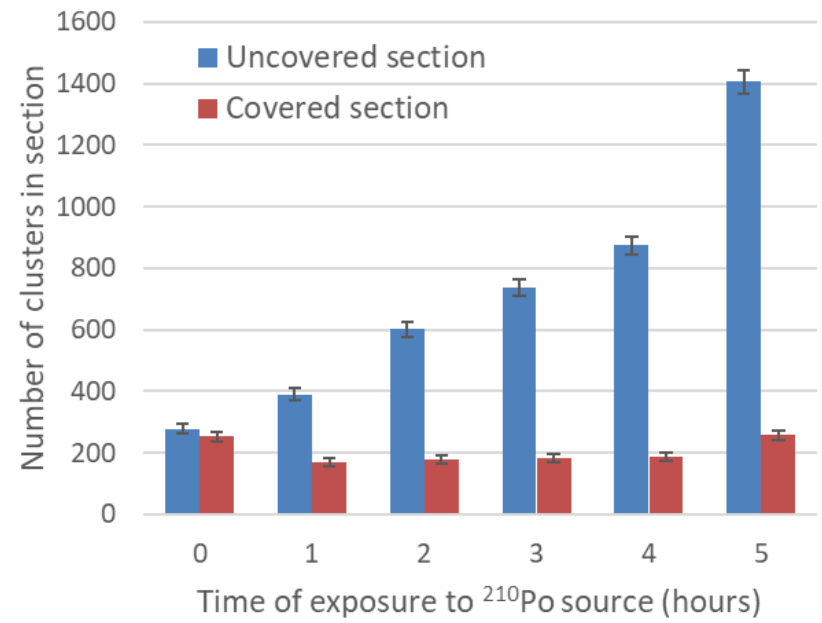

Fig. 4. The number of bright pixels in a 30-s background measurement for equivalent-size sections on the covered and uncovered side of the CCD after each additional hour of exposure to a ${ }^{210} \mathrm{Po}$ source.

\section{REPAIR MECHANISMS}

Multiple techniques have been considered to repair the damage produced by interactions with $\alpha$ radiation and to mitigate the effects of the damage during exposure. The techniques used have consisted of annealing, cooling and image processing. These may collectively be used to improve the accuracy of $\alpha$ detection and extend the useful lifetime of the device.

\section{A. Annealing}

Annealing was performed to repair some of the damage caused by the $\alpha$ radiation. The entire device was heated to a temperature of $100^{\circ} \mathrm{C}$ for 24 hours, reducing the amount of noise by $89 \%$ in one anneal. It has been found that the annealing is slightly less effective with each subsequent cycle, as in each case not all of the damage is repaired.

Heating to a higher temperature has been shown to be effective at repairing a greater amount of damage in other CCDs [5], however this would require dismantling the device due to the problems higher temperatures might cause, such as damaging electronic components. This would not be practical for standard use and so the $100^{\circ} \mathrm{C}$ temperature was used as this allows the entire device to be heated, while still resulting in significant reduction to the damage.

\section{B. Cooling}

To minimize the noise created by the damage during operation, the CCD is cooled. A copper heat pipe is used to draw heat away from the CCD and is then cooled by a fan. The noise reduction from cooling with different combinations of the fan and heat pipe can be seen in Fig. 5. The heat pipe alone is successful at reducing the noise by $56 \%$, which is useful for situations where it may not be practical to be operating a fan, such as where contamination may be in the form of dust. However, if a fan is also able to be used, the noise reduces by $97.5 \%$. This allows the CCD to be used for a much longer time between annealing, as although the rate of damage remains the same, it will not be as evident in the images produced.

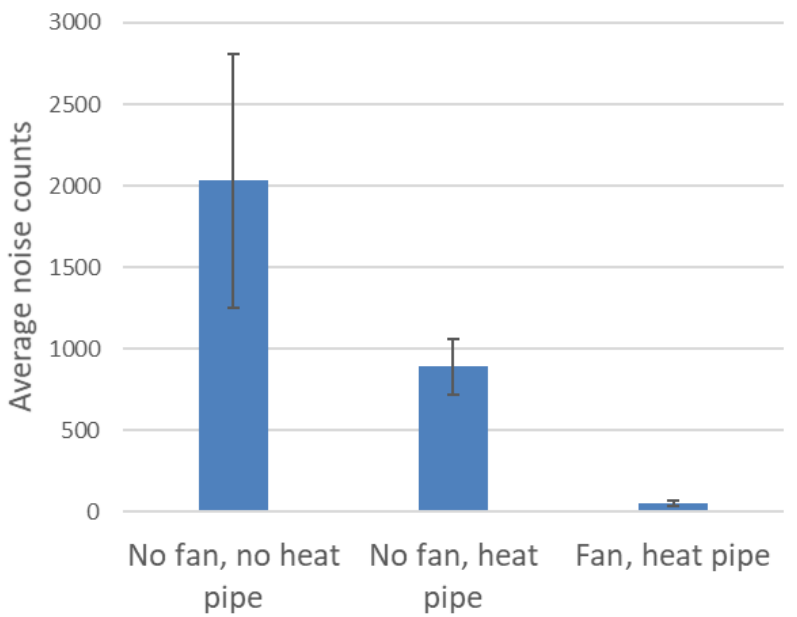

Fig. 5. The average number of bright pixels (noise) in images produced by the CCD in 5-minute exposures with no source present at three different levels of cooling: no cooling, just the heat pipe, and heat pipe with a fan.

\section{Image processing}

Any remaining noise can be removed by image processing. As the noise consists of pixels with lower intensity values than the pixels where $\alpha$ radiation interacted, and typically occurs in single pixels, the images can be processed to remove this low intensity noise. Images were taken with no CCD present to obtain pure noise images, and the average intensity of the pixels considered. This was compared with a typical $\alpha$ signal, to determine a cut-off intensity of 100 . For any cluster of pixels in which the brightest pixel has an intensity of less than 100, the entire cluster is removed. If any single pixel is over this threshold, the entire cluster is kept. This ensures that no information is lost from the $\alpha$-particle streaks, which are bright where the $\alpha$ particle interacted but tend to low intensities. An example section of an image before and after processing can be seen in Fig. 6. This shows the significant reduction in noise possible with image processing. 


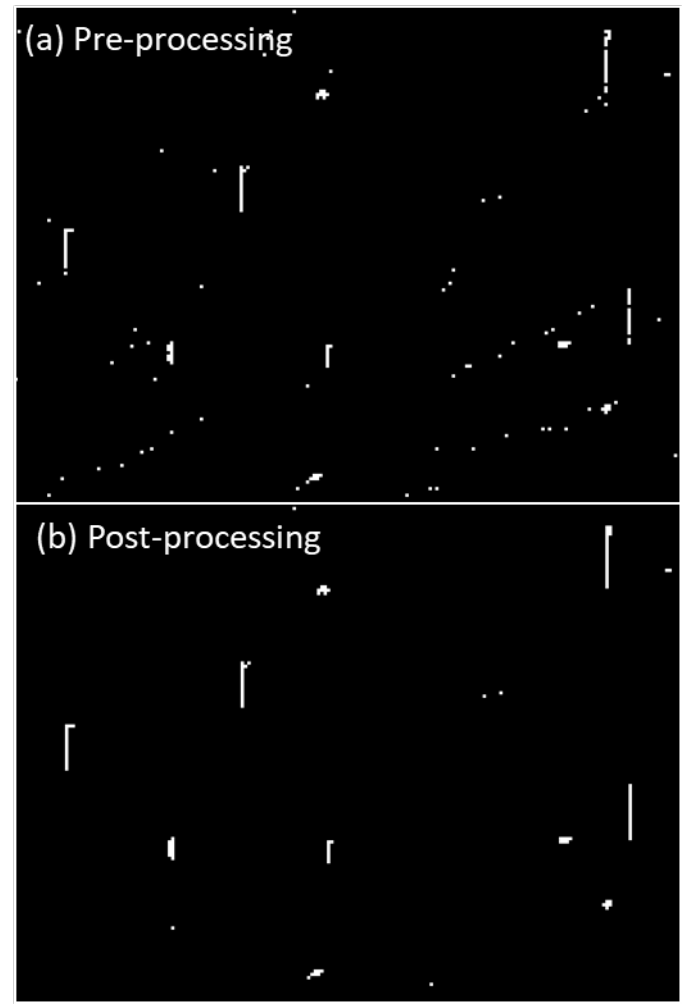

Fig. 6. A representative section of a CCD image produced under a 30-s exposure to a ${ }^{210} \mathrm{Po}$ source (a) before and (b) after processing to remove noise.

\section{CONCLUSION}

CCDs can be used to image and identify $\alpha$ radiation, however they are subject to damage from the $\alpha$ particles which produce increasing levels of noise over continued exposure. This noise can be reduced through cooling the device and image processing. As the noise builds to a problematic level, a significant amount of the damage can be repaired through annealing. By combining these techniques, the lifetime of the device as an $\alpha$-particle detector is greatly extended and the accuracy of detection improved by reducing false measurements or masked signals due to noise.

\section{REFERENCES}

[1] A. Aguilar-Arevalo et al., "Measurement of radioactive contamination in the high-resistivity silicon CCDs of the DAMIC experiment," J. Instrum., vol. 10, no. 08, pp. P08014-P08014, Aug. 2015.

[2] J. Estrada, J. Molina, J. J. Blostein, and G. Fernández, "Plasma effect in silicon charge coupled devices (CCDs)," Nucl. Instruments Methods Phys. Res. Sect. A Accel. Spectrometers, Detect. Assoc. Equip., vol. 665, pp. 90-93, 2011.

[3] R. Newton, J. Dykes, M. J. Scott, and M. J. Joyce, "Alpha contamination assay, dosimetry and spectrometry using charge coupled devices," 2016 IEEE Nucl. Sci. Symp. Med. Imaging Conf. Room-Temperature Semicond. Detect. Work. NSS/MIC/RTSD 2016, vol. 2017-Janua, 2017.

[4] R. Newton, M. J. Scott, and M. J. Joyce, "Investigating Artifacts Associated with beta-Particle Interactions in Charge-Coupled Devices," ... J. Mod. Phys. Conf. Ser., vol. 1, no. 1, pp. 1-5, 2010.

[5] G. R. Hopkinson and A. Mohammadzadeh, "Comparison of CCD Damage Due to 10- and 60-MeV Protons," IEEE Trans. Nucl. Sci., vol. 50, no. 6 I, pp. 1960-1967, 2003. 